\title{
Laser-wakefield accelerators for medical phase contrast imaging: Monte Carlo simulations and experimental studies
}

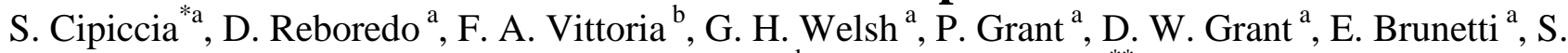
M. Wiggins ${ }^{a}$, A. Olivo ${ }^{b}$, D. A. Jaroszynski ${ }^{* * a}$

a Department of Physics, University of Strathclyde, 107 Rottenrow East, Glasgow, G4 0NG, UK; ${ }^{\mathrm{b}}$ Department Medical Physics and Biomedical Engineering, University College London, Gower

Street, London, WC1E 6BT, UK

\begin{abstract}
$\mathrm{X}$-ray phase contrast imaging (X-PCi) is a very promising method of dramatically enhancing the contrast of X-ray images of microscopic weakly absorbing objects and soft tissue, which may lead to significant advancement in medical imaging with high-resolution and low-dose. The interest in X-PCi is giving rise to a demand for effective simulation methods. Monte Carlo codes have been proved a valuable tool for studying X-PCi including coherent effects.

The laser-plasma wakefield accelerators (LWFA) is a very compact particle accelerator that uses plasma as an accelerating medium. Accelerating gradient in excess of $1 \mathrm{GV} / \mathrm{cm}$ can be obtained, which makes them over a thousand times more compact than conventional accelerators. LWFA are also sources of brilliant betatron radiation, which are promising for applications including medical imaging.

We present a study that explores the potential of LWFA-based betatron sources for medical X-PCi and investigate its resolution limit using numerical simulations based on the FLUKA Monte Carlo code, and present preliminary experimental results.
\end{abstract}

Keywords: X-ray, Phase-contrast imaging, betatron radiation, laser-plasma wakefield accelerator

\section{INTRODUCTION}

In a $L W F A^{1}$, a high intensity ultra-short duration laser pulse $\left(I>10^{18} \mathrm{Wcm}^{-2}\right)$ passing through plasma generates plasma density waves. The ponderomotive force associated with the light pressure expels electrons from the laser axis, while leaving behind the heavier ions. The expelled electrons experience the electrostatic restoring force of the quasi-stationary ions and start oscillating about the laser axis to produce the plasma density wake. If the laser pulse length is shorter than the plasma wavelength the wake structure assumes a bubble-like shape ${ }^{2}$. In the highly non-linear bubble regime the background electrons expelled from the laser axis can be trapped at the rear of the bubble and be accelerated. The accelerating gradient is in excess of $100 \mathrm{GeV} / \mathrm{m}^{3-5}, 1000$ times larger than in conventional radio-frequency cavities, which provides a new paradigm for table-top high energy accelerators.

The trapped electrons enter the bubble with a finite transverse momentum undergo transverse oscillations while they accelerate longitudinally, while they emitting synchrotron-like X-ray betatron radiation (Figure 1). The critical energy of the betatron radiation is tuneable from few $\mathrm{keV}$ up to hundreds of $\mathrm{keV}^{6,7}$. Radiation is emitted in duration $(\sim 10 \mathrm{fs})$ pulses from a small sizes source $\left(\sim\right.$ few $\left.\mu \mathrm{m}^{3}\right)$, which results in a peak brilliance of the order of $10^{23}$ photons $/ \mathrm{s} \mathrm{mrad}^{2} \mathrm{~mm}^{2} 0.1 \%$ B.W., which is comparable with the brilliance of third generation storage rings, but in a spectral range not accessible to synchrotrons. The compactness of the source makes the LWFA driven betatron radiation particularly appealing for applications such as X-PCi.

Conventional X-ray imaging based on the absorption is not suitable for weakly absorbing materials since the contrast is usually very low. X-PCi, on the other hand, relies on the phase front distortion introduced by the object, which enhances the contrast, which is extremely promising for medical applications where there is demand for high contrast, high resolution, low dose imaging.

The combination of X-PCi technique and a LWFA driven X-ray source offers a valuable compact tool for high resolution, high-contrast X-ray imaging. In Section 2 we show the characterization of the betatron source driven by the

*silvia.cipiccia@strath.ac.uk

**d.a.jaroszynski@strath.ac.uk 
LWFA of the ALPHA-X laboratory at the University of Strathclyde and a proof-of-principle X-PCi image of a common spider obtained in free space propagation. In Section 3 we model the betatron source using FLUKA ${ }^{8}$ Monte Carlo code and numerically investigate the resolution limits. In Section 4 we discuss the results and possible further improvement using the Edge-Illumination (EI) X-PCi technique?

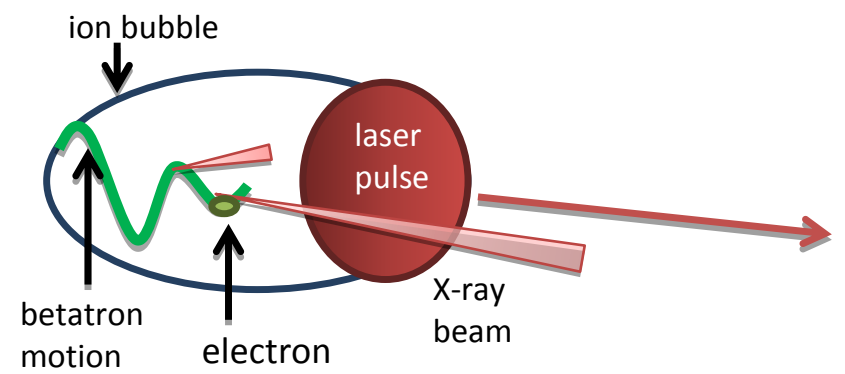

Figure 1: Schematic of the betatron emission process. The laser pulse generates a positively charged bubble structure that follows the laser pulse. The electrons injected at the rear of the bubble and wiggle around the laser axis while emitting x-ray radiation.

\section{LWFA BETATRON SOURCE CHARACTERIZATION}

The experiment was performed using the ALPHA-X laser wakefield accelerator at the Terahertz to Optical Pulse Source (TOPS) facility at the University of Strathclyde. $800 \mathrm{~nm}, 0.9 \mathrm{~J}$ and $35 \mathrm{fs}$ laser pulses from a Ti:sapphire laser are focused using a F/18 spherical mirror to a spot size of $40 \mu \mathrm{m}\left(1 / e^{2}\right)$, with an intensity $I=2 \times 10^{18} \mathrm{~W} / \mathrm{cm}^{2}$ on target at the entrance of a $2.8 \mathrm{~mm}$ diameter pulsed supersonic Helium gas jet to produce a $10 \mu \mathrm{m}$ relativistic plasma channel. A plasma density of $\sim 2.0 \times 10^{19}$ electrons $/ \mathrm{cm}^{3}$ was estimated characterizing the gas-jet using a Mach-Zehnder interferometer.

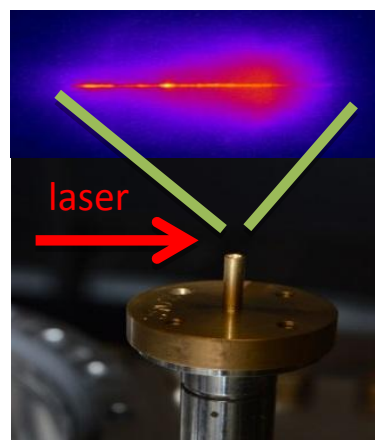

Figure 2: The TW laser pulse is focused at the entrance of the Helium gas-jet. The plasma channel produced is monitored by observing Thomson side scattering (gas-jet picture courtesy of Mr. Constantin Aniculaesei).

The emerging electron beam energy is monitored by dispersing the beam toward a scintillator Lanex (KODAK) phosphor screen with a bending magnet placed just after the gas-jet. Stable quasi-monoenergetic electron bunches with charge of 5-10 pC and an energy of $\approx 150 \mathrm{MeV}$ are measured. The accelerator is run at a repetition rate of $0.33 \mathrm{~Hz}$.

To measure the critical energy of the betatron radiation, the X-ray beam is passed through sets of metal filters $(\mathrm{Cu}, \mathrm{Al}, \mathrm{Ti}$ and $\mathrm{Ag}$ ). The shadows cast by the metal filters are recorded using Fuji BAS-SR imaging plates. The critical energy can be estimated from the attenuation of the X-ray beam. All measurements are performed in a single shot. The average critical energy estimated for the run is $\sim 15 \mathrm{keV}$ (Figure 3-a). The spatial distribution and the divergence of the X-ray beam is directly measured by imaging the full X-ray beam with imaging plates, giving an average divergence of $\sim 40$ mrad (Figure 3-b). 

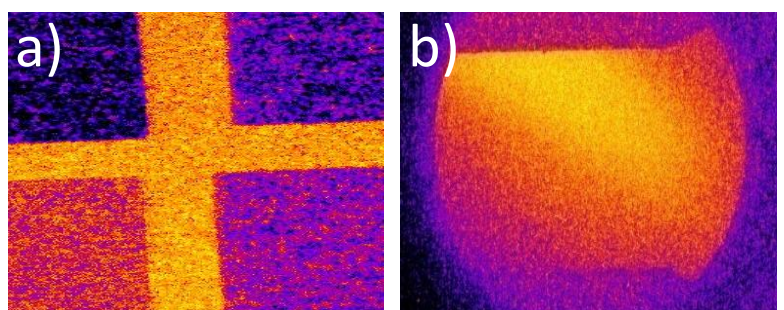

Figure 3: a) Shadow cast by the set of metal filter. From the top left, clockwise: $\mathrm{Cu} 0.1 \mathrm{~mm}$, Ti $0.1 \mathrm{~mm}, \mathrm{Ag} 0.05 \mathrm{~mm}, \mathrm{Al} 0.5 \mathrm{~mm}$. b) xray beam profile

As a proof of principle of the viability of using LWFA driven betatron radiation for X-PCi, a phase contrast experiment in free space propagation has been carried out using the X-ray beam produced by the ALPHA-X LWFA. Similar experiments have been performed using a more powerful laser and different source-sample and sample- detector distances $^{7}$. For the experiment a biological sample (a dead common arachnid) is placed a distance $R_{1}=0.82 \mathrm{~m}$ from the source. The phase contrast image is recorded using an image plate placed at $R_{2}=4.39 \mathrm{~m}$ from the object, which gives a magnification of $\mathrm{M}=\left(\mathrm{R}_{2}+\mathrm{R}_{1}\right) / \mathrm{R}_{1}=6.4$. The transverse coherent length is defined by $L=\lambda l / \sigma$, where $\lambda$ is the wavelength of the source, $l$ the source to observation distance and $\sigma$ the source size. For a $1 \mu \mathrm{m}$ source size, $15 \mathrm{keV} \mathrm{X-}$ rays and $5.2 \mathrm{~m}$ distance $\mathrm{L} \approx 400 \mu \mathrm{m}$. The image is recorded in 300 shots at a repetition rate of $0.33 \mathrm{~Hz}$. The resolution of the image is set by the imaging plate scanner to $50 \mu \mathrm{m}$, Figure 4.
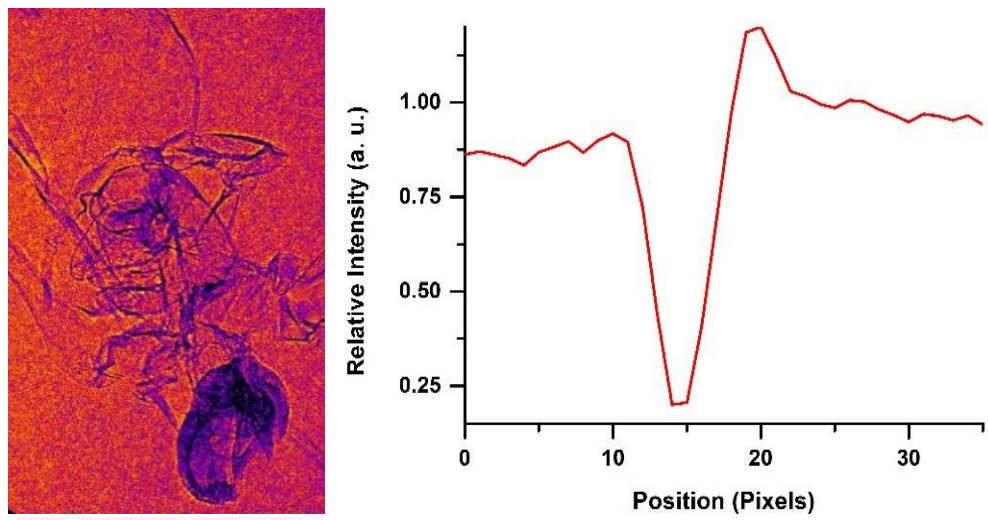

Figure 4: 1.h.s.: Free space propagation X-PCi of a common arachnid. r.h.s.: Relative intensity from the edge of a leg. Pixels size, 50 $\mu \mathrm{m}$

\section{MONTE CARLO SIMULATIONS}

Recently, Monte Carlo codes have been shown to be a valuable tool for numerically investigating the formation of phase contrast images, including either ray-tracing or the wave-optical approach for a full description of diffraction and interference phenomena ${ }^{10}$. We have used the FLUKA ${ }^{8}$ Monte Carlo code to model the LWFA based betatron source as a $15 \mu \mathrm{m}$ FWHM source with a $10 \mathrm{keV}$ critical energy. To investigate the potential of this source for X-PCi, we have used the ray-tracing approach to produce a phase contrast image of a $10 \mu \mathrm{m}$ bone crack in a $200 \mu \mathrm{m}$ thick slice. In the simulations the source-bone distance is $30 \mathrm{~cm}$, while 80,120 and $150 \mathrm{~cm}$ bone-detector distances are investigated. The simulation results are shown in Figure $\mathbf{5}$.

From FLUKA simulation results it appears that a $10 \mu \mathrm{m}$ bone crack will produce a detectable phase contrast image using a betatron source. Figure 6 shows the results of similar simulations for a $1 \mu \mathrm{m}$ crack. In this case, the phase contrast imaging is very faint, but still visible in Figure 6-a, but the contrast is washed out in Figure 6-b, when convoluting the image with the $13 \mu \mathrm{m}$ detector pixel size. 


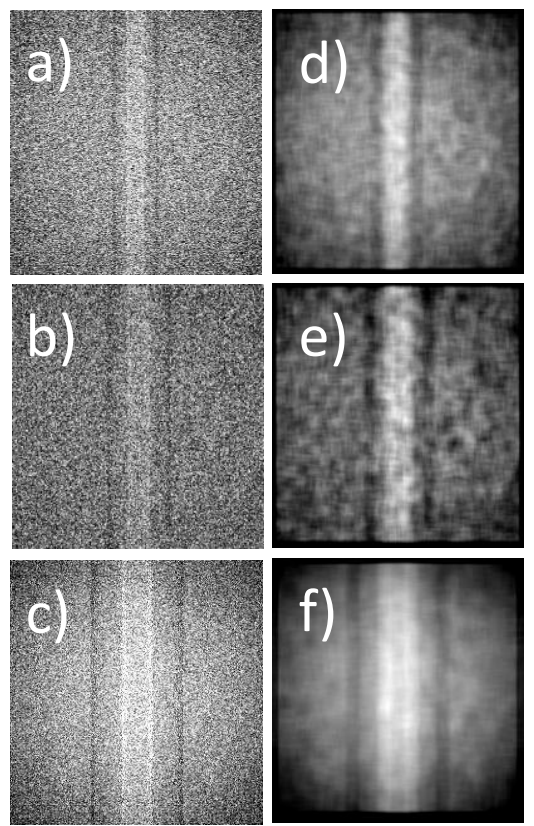

Figure 5: Phase contrast imaging of a $10 \mu \mathrm{m}$ crack in a $200 \mu \mathrm{m}$ thick slice. The source-bone distance is $30 \mathrm{~cm}$. The bone-detector distance is a) $80 \mathrm{~cm} \mathrm{b)} 120 \mathrm{~cm}$ and c) $150 \mathrm{~cm}$. The images d), e) and f) are obtained by convoluting a), b) and c) respectively with a 13 $\mu \mathrm{m}$ detector pixel size.
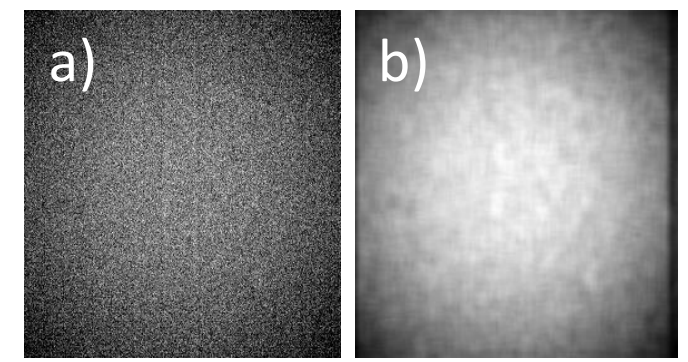

Figure 6: a) Phase contrast imaging of $1 \mu \mathrm{m}$ crack in a $200 \mu \mathrm{m}$ thick bone. The source-bone distance is $30 \mathrm{~cm}$, the bone detector distance is $150 \mathrm{~cm}$. b) same image convoluted with the $13 \mu \mathrm{m}$ detector pixel size.

\section{CONCLUSIONS}

We have presented preliminary studies that show the potential of a LWFA driven betatron X-ray source for X-PCi. The betatron source based on the ALPHA-X LWFA has been characterized experimentally and used to produce a proof-ofprinciple X-PCi in free space propagation of a biological specimen. The modelling of the source shows its potential for imaging cracks in bones on the order of $10 \mu \mathrm{m}$. The imaging fails when the crack size is reduced to $1 \mu \mathrm{m}$. A possible solution to the problem is to use the Edge Illumination (EI) X-PCi technique ${ }^{9,11}$. The EI setup is based on a system of two masks placed respectively before the object and in front of the detector. The image is reconstructed by scanning the object and the resolution is set by presample mask ${ }^{12}$. It is therefore possible, by combining the EI technique with the LWFA based betatron source, to image micron size crack with micrometre resolution.

\section{ACKNOWLEDGMENTS}

S.C., D.R., G.H.W., P.G., D.W.G., E.B., S.M.W. and D.A.J. acknowledge the support of the U.K. EPSRC (EP/J018171/1) and the EC's $7^{\text {th }}$ Framework Programme (LASERLAB-EUROPE no. 284464, the EUCARD-2 project (no. 312453) and the Extreme Light Infrastructure (ELI) project). A.O. and F.A.V. are supported by the EPSRC (EP/I021884/1 and EP/I022562/1). 


\section{REFERENCES}

[1] Tajima, T. \& Dawson, J. M. "Laser electron accelerator". Phys. Rev. Lett. 43, 267 (1979).

[2] Pukhov, A. \& Meyer-Ter-Vehn, J. "Laser wake field acceleration: the highly non-linear broken wave regime". Appl. Phys. B 74, 355-361 (2002).

[3] Mangles, S. P. D. \& al., e. "Electron Acceleration from the Breaking of Relativistic Plasma Waves". Nature 431, 535-538 (2004).

[4] Geddes, C. G. R. \& al., e. "High Quality Electron Beams from a Laser Wakefield Accelerator Using PlasmaChannel Guiding". Nature 431, 538-541 (2004).

[5] Faure, J. \& al., e. "A Laser-Plasma Accelerator Producing Monoenergetic Electron Beams". Nature 431, 541544 (2004).

[6] Kneip, S. et al. "Bright Spatially Coherent Synchrotron X-rays from a Table-top Source". Nat Phys 6, 980-983 (2010).

[7] Cipiccia, S. et al. "Gamma-Rays from Harmonically Resonant Betatron Oscillations in a Plasma Wake". Nat Phys 7, 867-871 (2011).

[8] Battistoni, G. et al. "The FLUKA code: description and benchmarking". Hadronic Shower Simulation Workshop 896, 31-49 (2007).

[9] Olivo, A. \& Speller, R. "A coded-aperture technique allowing X-ray phase contrast imaging with conventional sources". Appl Phys Lett 91, 074106 (2007).

[10] Cipiccia, S., Vittoria, F. A., Weikum, M., Olivo, A. \& Jaroszynski, D. A. "Inclusion of coherence in Monte Carlo models for simulation of X-ray phase contrast imaging". Opt Express 22, 23480-23488 (2014).

[11] Diemoz, P. C. et al. "X-Ray Phase-Contrast Imaging with Nanoradian Angular Resolution". Phys Rev Lett 110 , 138105 (2013).

[12] Diemoz, P. C., Vittoria, F. A. \& Olivo, A. "Spatial resolution of edge illumination X-ray phase-contrast imaging". Opt. Express 22, 15514-15529 (2014). 